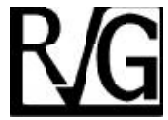

\title{
Costes y precios en los contratos con el Ministerio de Defensa de España: caso de estudio
}

\author{
Aguado Romero, José* \\ López Hernández, Antonio Manuel ${ }^{* *}$ \\ Vera Ríos, Simón ${ }^{\star \star \star}$
}

\section{Resumen}

La importancia económica de las adquisiciones de defensa mediante contratos no competitivos ha motivado que los departamentos de defensa españoles dicten normas específicas para el control de costes y precios de dichos contratos. El objetivo de este artículo es analizar las distintas fases del proceso de cálculo de los componentes de los contratos con el Ministerio de Defensa de España. En el marco de la teoría institucional, utilizando una metodología de carácter cualitativo, en concreto, mediante el método del caso, se analiza el sistema de costes implantado por la multinacional Indra Sistemas, S.A. para adecuarse al marco normativo establecido por el Ministerio de Defensa. Los resultados muestran que dicho sistema responde a un enfoque de asignación de costes completos y representa un modelo derivado de la aplicación a Indra de la normativa española sobre el cálculo de costes en los contratos de defensa. Se concluye que la normativa española sobre cálculo de costes y auditoría de contratos de defensa está influyendo en los sistemas de contabilidad de gestión de los contratistas.

Palabras clave: Contabilidad de gestión, normas de costes, contratistas de defensa, Ministerio de Defensa de España, Indra.

\section{Recibido: 15-06-12. Aceptado: 27-10-13}

* Interventor del Ministerio de Defensa, Profesor Asociado de la Universidad de Granada (España). Doctor en Ciencias Económicas y Empresariales por la Universidad de Granada. E-mail: jaguado@ugr.es

** Presidente de la Cámara de Cuentas de Andalucía. Catedrático de Universidad (España), adscrito al Departamento de Economía Financiera y Contabilidad de la Universidad de Granada. E-mail: alopezh@ugr.es

*** Profesor Titular de la Universidad de Granada (España), en el Departamento de Economía Financiera y Contabilidad. Doctor en Ciencias Económicas y Empresariales y Licenciado en Psicología. E-mail: svera@ugr.es 


\section{Costs and Prices for Contracts with the Defense Ministry of Spain: Case Study}

\section{Abstract}

The economic importance of defense acquisitions through non-competitive contracts have motivated Spanish defense departments to dictate specific standards to control costs and prices for these contracts. The objective of this article is to analyze the different phases in the process of calculating components for contracts with the Defense Ministry of Spain. Within the framework of institutional theory, utilizing qualitative methodology, specifically, a case study, this work analyzes the cost system implanted by the multinational Indra Sistemas, S.A., to adapt itself to the framework of standards established by the Defense Ministry. Results show that the aforesaid system follows an approach of assigning complete costs and represents a model derived from applying the Spanish norms regarding cost calculation in defense contracts to Indra. Conclusions are that the Spanish norms on cost calculation and defense contract auditing are influencing management accounting systems for the contractors.

Key words: Management accounting, cost standards, defense contractors, Defense Ministry of Spain, Indra.

\section{Introducción}

En el contexto de las adquisiciones de bienes y servicios específicos para la defensa, se observa una problemática muy similar en los países desarrollados derivada de la existencia de un mercado imperfecto (Aguado y Zafra, 2011; Currie, 2011). El mercado de defensa se caracteriza por el oligopolio de la oferta de los sistemas de armas y equipos militares, y el monopsonio de la demanda de los bienes y equipos militares, siendo los principales clientes los departamentos de defensa en sus respectivos países (Gil, 2002; Fonfría y Correa, 2010).

A pesar de las restricciones industriales, económicas y políticas existentes en el mercado de defensa y la situación de crisis económica actual, las cifras del gasto militar en el mundo (1.738 billones de dólares en 2011) muestran la impor- tancia económica y el impacto de las adquisiciones de material militar, muy vinculadas a los presupuestos de defensa, en las economías nacionales.

Particularmente América Latina fue la región mundial con mayor aumento de gasto bélico en 2010 , con un incremento de 5,1\% (destacando los aumentos en Brasil, Chile, Colombia y Perú). Sin embargo, en 2011 la región registró un descenso de 3,3\% en el gasto militar en términos reales. En Europa la caída fue de 1,9\% en el año 2011 como resultado de las medidas de austeridad adoptadas (Stockholm International Peace Research Institute, 2012).

En el contexto internacional y dadas las peculiaridades que confluyen en los bienes y equipos militares, existen razones políticas, económicas (mercado imperfecto, defensa de la industria nacional), tecnológicas (alta especificidad, fa- 
bricación de series cortas, alta demanda de requerimientos tecnológicos, ciclos de vida amplios, entre otras) y de seguridad nacional (factores estratégicos) que han motivado a los departamentos de defensa a satisfacer sus demandas a través de contratos adjudicados a una empresa concreta mediante procedimientos negociados, y no mediante importaciones o licitaciones abiertas.

En este marco y con el fin de suplir los mecanismos de mercado y las asimetrías de la información (Kirat et al, 2003) que sufren los departamentos de defensa frente a los contratistas, se han desarrollado en los principales países de la Organización del Tratado del Atlántico Norte (OTAN) procedimientos y análisis alternativos, tales como técnicas de evaluación de costes y precios de los contratos. Estos análisis permiten determinar la razonabilidad de los precios de los contratos adjudicados mediante procedimientos negociados, garantizando la aplicación eficiente de los recursos públicos invertidos en defensa.

Para el empleo de estas técnicas, la contabilidad de la empresa y los estudios técnico-industriales se configuran como los soportes necesarios para evaluar, mediante procedimientos normalizados, si el precio ofertado por un contratista es razonable $o$ adecuado al mercado. Mediante esta revisión o evaluación, los departamentos de defensa obtienen información que les permite comprobar si están pagando ineficacias técnicas de la empresa, un sobreprecio derivado de costes no registrados o no admitidos, o unos márgenes de beneficio que, más allá de obedecer a los requerimientos 0 incertidumbres en la ejecución del contra- to, responden a una posición casi monopolística de la empresa contratista en el mercado (Rogerson, 1989; Smith, 1990).

Sin embargo, para responder a los requerimientos de los organismos auditores de los departamentos de defensa encargados de la revisión y el control de los costes y precios de los contratos, las empresas que deben someterse a dichas revisiones se han visto obligadas a reconsiderar y adaptar su sistema de contabilidad de gestión para adecuarlo a una normativa cuyos planteamientos y criterios no siempre resultan coincidentes con los implantados en las mismas (Aguado, 2009).

El objetivo general de este artículo es analizar las distintas fases del proceso de cálculo de los componentes de los contratos establecidos entre el Ministerio de Defensa (MINISDEF) y una empresa determinada, sujeta tanto al cumplimiento de una normativa específica sobre costes como a rigurosos procesos de revisión de precios, con. A tal efecto, se presenta un estudio empírico centrado en una Indra Sistemas S.A. (Indra), empresa representativa del sector de la defensa, para analizar la incidencia en los procesos formativos del coste y del precio de las ofertas realizadas por los contratistas, de la normativa del MINISDEF sobre auditoría de contratos y cálculo de costes en los contratos no competitivos.

Indra es la multinacional de tecnologías de la información número uno en España y una de las principales de Europa y Latinoamérica, contando entre sus clientes a diferentes ejércitos de países europeos y latinoamericanos. Las razones que llevaron a la elección de Indra como empresa objeto del presente estudio fueron, su importancia estratégica 
para la defensa nacional de España ${ }^{1}$, su alto volumen de contrataciones con el MINISDEF, tanto en número de contratos como en su cuantía económica, así como el hecho de haber sido la empresa más auditada por el Grupo de Evaluación de Costes (GEC) del MINISDEF.

Dentro del contexto de la investigación en contabilidad de gestión son numerosos los autores que han planteado la necesidad de las organizaciones de adaptar sus sistemas de información al entorno en el que desarrollan su actividad, pues en caso contrario, no podrán ser competidores globales, eficientes y efectivos (Al-Omiri y Drury, 2007; Bescos, 2003; Selto y Widener, 2004).

Este trabajo se fundamenta en la teoría institucional ya que ésta presenta dos aplicaciones básicas adecuadas a la investigación: proporciona una estructura para explicar el proceso de legitimación llevado a cabo por la organización analizada en un contexto industrial, económico y político concreto (Scott, 1995; Scapens, 1994; Escobar y Lobo, 2002), y sirve de base para justificar la adopción de determinados patrones de cambio en contabilidad de gestión. Según el enfoque institucional, el Estado se considera un actor central en la explicación de las características de las organizaciones debido a las presiones coercitivas que puede ejercer mediante la fuerza, la persuasión, la legislación, entre otros (Abzug y Mezias, 1993).
En el campo de la contabilidad de gestión los estudios de Covaleski et al (1993) y Carmona et al (1998) analizaron cómo las organizaciones introducen cambios en sus sistemas de contabilidad de gestión, buscando legitimar sus acciones en el entorno (Suchman, 1995) ante las presiones institucionales externas más que por razones de eficiencia. Ejemplos de estudios del cambio contable bajo la teoría institucional son analizados en Burns (2000), Granlund (2001), Carruthers (1995) y Carmona y Macías (2001), donde exploran cómo y por qué se adoptaron nuevas prácticas contables.

El presente artículo se inserta en un trabajo de investigación mucho más amplio realizado sobre la auditoría de costes y precios en España. En dicha investigación se analiza tanto la evolución de la metodología y los procedimientos de trabajo empleados por el MINISDEF, como la repercusión que el ejercicio de dichas auditorías tienen sobre las empresas auditadas en una doble dirección: por un lado, su influencia en los sistemas de información para la gestión de las empresas y, por otro, los efectos económicos derivados de las auditorías de costes y precios en términos de ajustes sobre los precios de los contratos de defensa.

Este trabajo se desarrolló a través de una metodología de carácter cualitativo. Se seleccionó el estudio de caso como método de investigación teniendo 
en cuenta las características del fenómeno objeto de estudio, el objetivo perseguido y las circunstancias que concurren en las empresas adjudicatarias de contratos del MINISDEF. Este método proporciona un conocimiento de la práctica contable de gestión y permite estudiar el proceso de cambio contable dentro del contexto en que se produce (Araujo, 2003; Granlund, 2001; Yin, 2003). En estudio de caso se realiza un análisis descriptivo y explicativo, en el cual se pretende argumentar las razones de las prácticas contables puestas en funcionamiento en la empresa motivadas por sus relaciones contractuales con el MINISDEF.

La estructura del control del MINISDEF sobre los contratos de defensa se basa fundamentalmente en dos pilares: la normativa reguladora de la auditoría de contratos y el órgano auditor que realiza las evaluaciones de costes y precios. La normativa reguladora de la auditoría de contratos se contiene en dos disposiciones: la Orden Ministerial 238/1998 (Ministerio de Defensa, 1998) sobre la presentación y auditoría de ofertas y normas sobre cálculo de costes en ciertos contratos del MINISDEF y la Instrucción 128/2007 (Secretaría de Estado de Defensa, 2007) sobre los procedimientos para la prestación de los servicios de análisis de costes y precios en el ámbito del MINISDEF.

Para comprobar que las empresas cumplen con los requerimientos del MINISDEF se ha impuesto a las empresas una cláusula específica en los contratos por la que están sujetas a someterse a los procesos de evaluación de costes y precios sobre los contratos en cuestión. Esta evaluación la realiza el GEC a instancia de los órganos de contratación del MINISDEF, mediante técnicas de auditoría y/o análisis.

La auditoría de contratos de defensa en España se aplica a aquellos contratos de suministros de fabricación, de consultoría y asistencia y de los de servicios que se adjudiquen por el procedimiento negociado sin publicidad, y cuyo presupuesto sea igual o superior a $901.518,16$ $€ \mathrm{y}$, opcionalmente, a los de presupuesto inferior a dicho importe.

El Grupo de Evaluación de Costes situado orgánicamente bajo la dependencia de la Dirección General de Asuntos Económicos del MINISDEF, es el órgano encargado dentro del Departamento de efectuar el análisis de costes y precios de las empresas suministradoras o que participen en programas de defensa. EI GEC para la revisión de los costes y precios de los contratos aplica principalmente las Normas sobre los Criterios a emplear en el Cálculo de Costes en determinados contratos de suministros, consultoría y servicios del Ministerio de Defensa (NODECOS) que se encuentran reguladas en el anexo de la Orden Ministerial 283/98 (Ministerio de Defensa, 1998).

\section{La configuración del precio de los contratos con el MINISDEF}

Indra, para dar cumplimiento a lo establecido en las NODECOS (MINISDEF, 1998) sobre la elaboración y presentación de ofertas y, suministrar información para la auditoría de contratos en 
el "mercado"2 de Defensa y de Simuladores y Sistemas Automáticos de Mantenimiento (SIMSAM), además de calcular los costes directos de cada proyecto, calcula los recargos imputables al mismo en concepto de costes indirectos (de fabricación, de manipulación de materiales y de gastos generales y administración). De esta manera, en la estructura del precio de una oferta o contrato se diferencian cuatro componentes: costes directos, costes indirectos, costes financieros y beneficio aplicable al proyecto. La adaptación realizada por Indra en su contabilidad de gestión para elaborar y presentar los precios de las ofertas al MINISDEF es analizada en el presente trabajo, prestando especial atención a la formación de los cuatro componentes del precio citados.

Como presentación y guía del proceso de formación del coste y, en definitiva, del precio de las ofertas que elabora Indra para los órganos de contratación del MINISDEF, se exponen en el Cuadro 1 su estructura y componentes. Dicha estructura representa una adaptación de la empresa a lo dispuesto en la Instrucción 128/2007 (Secretaria de Estado de Defensa, 2007) sobre elaboración de ofertas por parte de los contratistas.

Este estudio se centra en los proyectos calificados como productivos o "facturables", es decir, proyectos de ventas a terceros ajenos a la empresa que están soportados por contratos entre Indra y el MINIS-
DEF adjudicados mediante procedimiento negociado o diálogo competitivo.

El proceso de cálculo y asignación de costes comienza en Indra cuando los factores disponibles se aplican al proceso de producción. En este momento se establece una relación entre las cuentas de la contabilidad financiera y los componentes de costes creada por Indra en su contabilidad de gestión, clasificando de esta forma los costes según su naturaleza (materias primas y otros aprovisionamientos, servicios exteriores, personal, financieros, amortizaciones y provisiones). Este proceso de conexión entre elementos de la contabilidad financiera y la contabilidad de gestión (Diagrama 1) se realiza teniendo en cuenta dos circunstancias: a) una cuenta solamente puede tener un componente de coste, y b) un componente de coste puede tener varias cuentas.

Los costes en los contratos con el MINISDEF, según lo establecido en la norma primera ${ }^{3}$, duodécima y decimotercera de las NODECOS (MINISDEF, 1998) se clasifican en "costes admisibles" y "costes no admisibles". Para determinar si un coste es admisible o no, se utilizan como criterios varios parámetros contenidos en la norma primera: su razonabilidad, su imputabilidad, su adecuación a las NODECOS (MINISDEF, 1998) y a las prácticas y principios de contabilidad de gestión generalmente aceptados, así como su concordancia con las disposiciones del contrato.

2 Unidades de negocio de la empresa Indra.

3 La norma primera de las NODECOS (MINISDEF, 1998) señala: "El coste total de un contrato está constituido por la suma de los costes admisibles, directos e indirectos, afectables o imputables al contrato". 


\section{Cuadro 1 \\ Cálculo de costes y precio de la oferta en Indra}

+ Costes Directos:

+ Costes Indirectos:

$=$ Coste Total

+ Beneficio

= Precio de la Oferta Antes de Impuestos

+ Impuestos (IVA)

= Precio de La oferta

Fuente: Elaboración propia a partir de la estructura de oferta contenida en la Instrucción 128/2007 (Secretaria de Estado de la Defensa, 2007) y de la información contenida en Indra (2004 y 2006).

\section{Diagrama 1 \\ Costes según su naturaleza}

\section{Contabilidad Financiera}

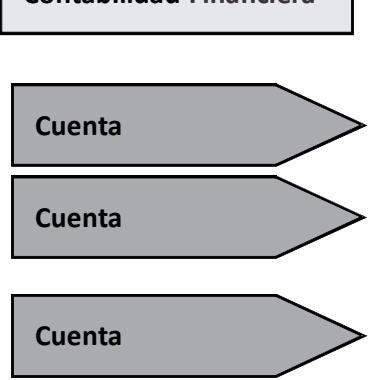

\section{Cuenta}

Mano de obra, materiales y subcontratación, otros costes directos

Recargos por costes indirectos de gestión (Overhead, Handling, G\&A) Recargos Financieros (Costes financieros del inmovilizado afecto a laactividad productiva, Periodo Medio de Maduración,Periodo Medio de Cobro)

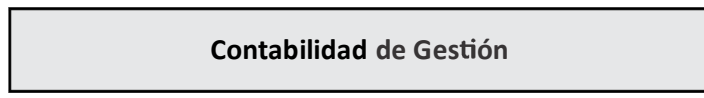

Fuente: Indra (2006).

Por su parte, la norma duodécima contempla el tratamiento específico y más detallado de diversos costes a efectos de su consideración como costes admisibles, o bien, como no admisibles.

Indra en el proceso de adaptación a las NODECOS (MINISDEF, 1998) procedió a diferenciar en su sistema de contabilidad de gestión entre ambos tipos de costes y para ello distingue, en los denominados "componentes de coste" de su contabilidad de gestión, entre "admisibles" y "no admisibles" (Cuadro 2) según su adecuación a lo dispuesto en las NODECOS (MINISDEF, 1998). No obstante, también en este proceso la empresa se ha adaptado a los criterios sobre esta clasificación dictados por el GEC y la Defense 


\section{Cuadro 2}

Componentes de coste no admisibles en Indra

\begin{tabular}{|c|c|c|c|c|c|}
\hline Código & Componente de Coste & $\%$ & Código & Componente de Coste & $\%$ \\
\hline 6200000 & $\begin{array}{l}\text { Gastos de investigación y } \\
\text { desarrollo del ejercicio }\end{array}$ & 100 & 6680000 & $\begin{array}{l}\text { Diferencias negativas de } \\
\text { cambio }\end{array}$ & 100 \\
\hline 6230029 & $\begin{array}{l}\text { Otros servicios } \\
\text { profesionales. Grupo Indra }\end{array}$ & 100 & 6690000 & Otros gastos financieros & 100 \\
\hline 6270001 & Publicidad & 50 & 6780000 & Gastos extraordinarios & 100 \\
\hline 6270002 & Ferias y exposiciones & 50 & 6781000 & Indemnizaciones & 100 \\
\hline 6271000 & Gastos de representación & 70 & 6811000 & Amortización de I+D & 100 \\
\hline 6300000 & Impuesto sobre beneficios & 100 & 6940000 & $\begin{array}{l}\text { Dotación provisión } \\
\text { insolvencias de tráfico }\end{array}$ & 100 \\
\hline 6490001 & $\begin{array}{l}\text { Ayuda de comidas origen } \\
\text { finanzas }\end{array}$ & 1,50 & 6950000 & $\begin{array}{l}\text { Dotación Provisión Otras } \\
\text { Operaciones de Tráfico }\end{array}$ & 100 \\
\hline 6940091 & Ayuda comida & 1,50 & 6960000 & $\begin{array}{l}\text { Provisión por depreciación } \\
\text { de valores }\end{array}$ & 100 \\
\hline 6620000 & Gastos financieros-Intereses & 100 & 7680000 & $\begin{array}{l}\text { Diferencias positivas de } \\
\text { cambio }\end{array}$ & 100 \\
\hline
\end{tabular}

Fuente: Elaboración propia a partir de información suministrada por Indra (2004).

Contract Audit Agency (DCAA), negociando con estos organismos su inclusión o no y extrapolando su consideración como admisible o no a ambos organismos auditores. De este modo, desde un punto de vista teórico, en la actualidad no existen discrepancias entre el GEC y la DCAA en la delimitación entre costes admisibles y no admisibles.

Una vez identificados los componentes de coste y agrupados según su naturaleza, en Indra el tipo de proyecto a que se destinen dichos componentes de coste determina si éste es directo o indirecto. Por tanto, los costes no se caracterizan a priori como directos o indirectos, dado que su adscripción a un tipo u otro depende del portador de coste, o sea, del proyecto. Si el componente de coste se destina a proyectos facturables, de ofer- tas o de Investigación y Desarrollo ( $(+D)$, se considera directo. En cambio, si el destino del componente de coste es un proyecto de estructura o interno se califica este componente de coste como indirecto respecto del proyecto facturable $y$ aparece como coste de estructura en la cuenta de resultados (Diagrama 2).

Para entender y valorar la adaptación de la empresa a las exigencias del MINISDEF en sus relaciones contractuales se debe tener presente que los sistemas de información contables de Indra, se han orientado tradicionalmente a la afectación de los costes directos, no siendo objeto de atención el cálculo de los costes indirectos. Indra utilizó con carácter general y para todos sus mercados el modelo de asignación de costes parciales, en concreto, en su modalidad de costes directos. Sin embargo, para adap- 


\section{Diagrama 2 \\ Costes según su función}

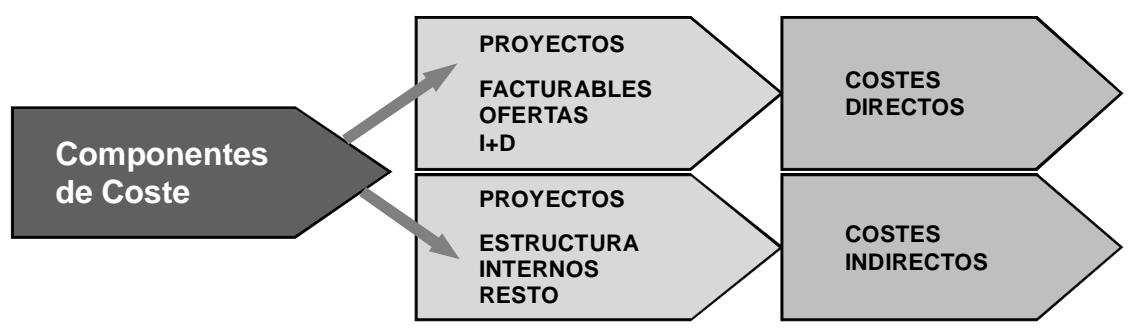

Fuente: Indra (2006).

tarse a las normas de costes del Ministerio de Defensa español, en el "mercado" de Defensa y de Simuladores y Sistemas Automáticos de Mantenimiento diseñó un modelo de asignación de costes completos. Este modelo de costes completo no fue extrapolado al resto de "mercados" de Indra.

En el Diagrama 3 se ofrece, a modo de presentación, un esquema general de la formación del coste de los contratos acordados por Indra con el MINISDEF. Como parte de nuestro estudio, el análisis de las distintas fases del proceso de cálculo de los componentes de los contratos con el MINISDEF se estudia de forma pormenorizada en los siguientes apartados de este trabajo.

\subsection{Los costes directos}

Un coste directo se define como aquél que no habría ocurrido si el contrato no hubiera existido (Arviso, 2006). La consideración y el concepto que de los costes directos manejados por Indra responden a lo establecido en las NODECOS (MINISDEF, 1998). Éstas, en su norma primera, definen los costes direc- tos como aquéllos vinculados a la actividad generada por la ejecución de un contrato, relacionando el consumo de factores con el proceso de producción y éste con el contrato, y facilitando el establecimiento con certeza de la medida técnica y económica del consumo.

En Indra todos los costes directos están recogidos en los proyectos del tipo facturables, de ofertas y de investigación y desarrollo. Éstos últimos se refieren a proyectos realizados por la empresa pero que no tienen correspondencia con ningún cliente o contrato, no generan ingresos. Son una necesidad estimada por Indra pero no soportada por un contrato con un cliente. En el caso de existir un contrato que ampare un proyecto de $\mathrm{I}+\mathrm{D}$ se considera un proyecto facturable. Al final del año la empresa lleva los costes directos de I+D a cuentas del inmovilizado y los activa posibilitando así su amortización. La norma undécima de las NODECOS (MINISDEF, 1998) permite recuperar los costes de I+D a través de los recargos.

En cuanto a los proyectos de ofertas, comprenden los costes de licitación y preparación de ofertas. Dentro de ellos 


\section{Diagrama 3 \\ Esquema de asignación de costes a los proyectos}

Clasificación

Clases de Costes
Localización

Unidades de empresa

Afectación

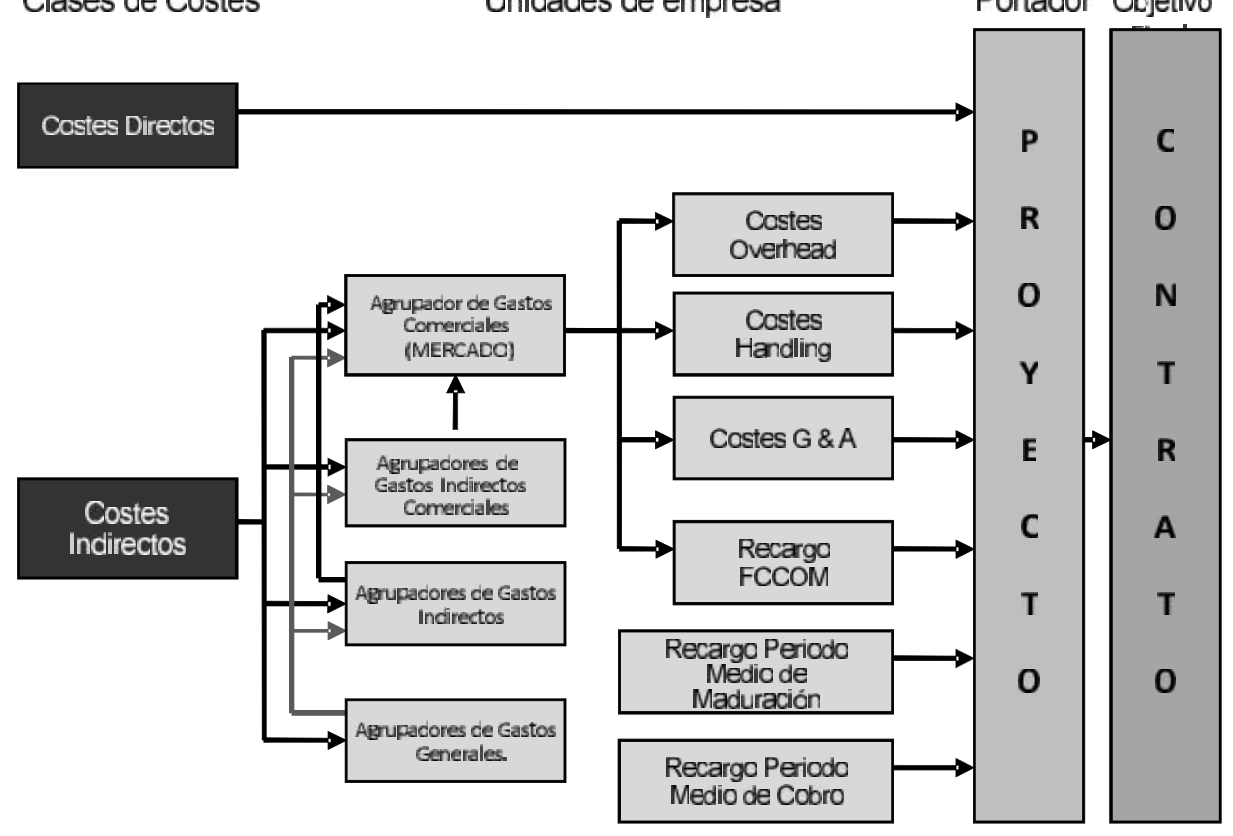

Fuente: Elaboración propia a partir de la información suministrada por Indra (2004 y 2006).

hay que distinguir entre las ofertas fallidas y las ofertas que no se han concretado en contratos a final de año. En el primer caso, se llevan a la cuenta de resultados del ejercicio y la vía para recuperar el coste directo de las ofertas es el recargo de gastos generales y administración. En el segundo caso, si aún el contrato no está adjudicado, y atendiendo a las circunstancias que concurran, el tratamiento contable del coste de dicha oferta puede ser diferente: se puede, como en el caso anterior, llevar al resultado del ejercicio, o bien considerarlo como una obra en curso o gasto pendiente de liquidar.
Posteriormente, si la empresa consigue la adjudicación del contrato, dichos costes pasan a considerarse costes directos de un proyecto facturable. En caso contrario, se debe proceder a llevar dichos costes a la cuenta de resultados.

En los proyectos que recogen costes directos, y con el objetivo de simplificar los informes requeridos por el GEC para la realización de sus auditorías, Indra creó códigos de control para agrupar, según su naturaleza, los componentes de coste atribuibles a estos tipos de proyectos. Los componentes de costes se agrupan en los códigos de control presentados en el Diagrama 4. 


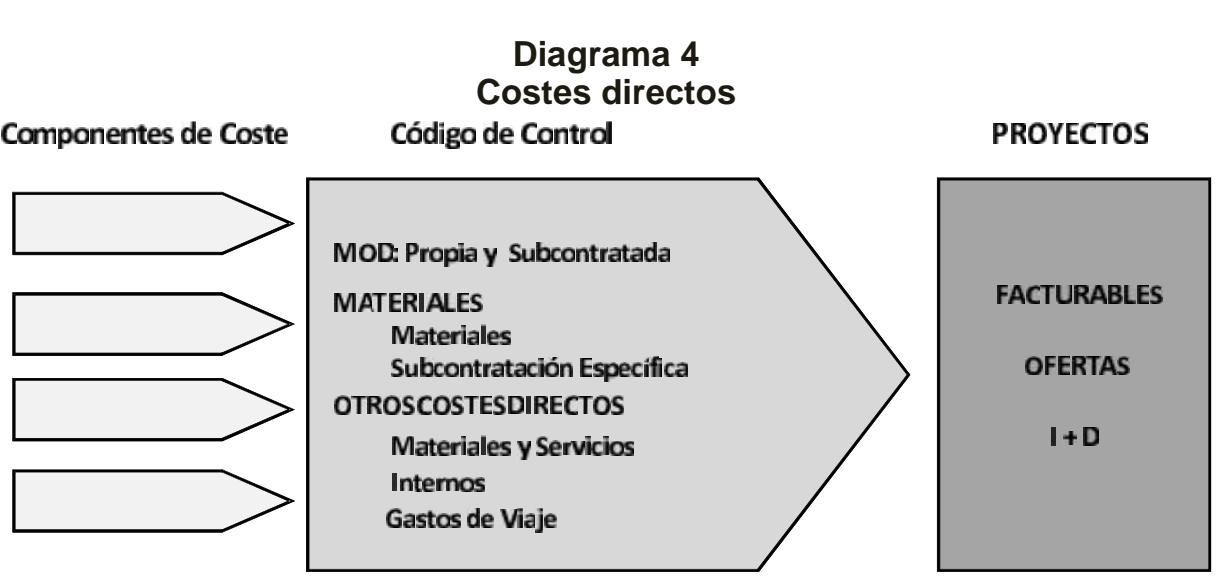

Fuente: Indra (2006).

Respecto a la mano de obra directa que imputable a los proyectos, la norma sexta de las NODECOS (MINISDEF, 1998) es la única que trata sobre esta clase de coste y en la misma se propugna la utilización de un coste estándar para ella. El instrumento clave para imputar el coste derivado de la participación de la mano de obra directa (propia y subcontratada) en los proyectos facturables de Indra es la tasa, cuya unidad de medida es la hora. El cálculo de la tasa horaria se efectúa por parte de Indra cada año para cada categoría profesional, siendo dichas tasas revisadas por el GEC.

El cálculo de la tasa se realiza sumando todos los conceptos salariales de los empleados (sueldos y salarios, incentivos, las primas por objetivos establecidas por la empresa, pluses, horas extraordinarias, ayuda a la comida, otros gastos sociales), más los costes de seguridad social, más los costes que facturan por el personal subcontratado (tasa horaria facturada por 1.742 horas $^{4}$ ). Una vez obtenida la suma anterior respecto a cada una de las categorías facturables indicadas, se divide entre las horas efectivas de trabajo de cada una, dando como resultado la tasa media a aplicar a la mano de obra directa durante el año.

Para efectuar el cálculo de las horas efectivas de trabajo a considerar, se parte de las horas de producción teóricas establecidas durante un año, según lo pactado en los respectivos convenios colectivos, de las cuales se restan las horas de improductividad del personal propio (formación, absentismo, enfermedad y otros), y a la cifra resultante se le suma el resultado de multiplicar 1.742 horas por el número de subcontratados. 


\subsection{Los costes indirectos: proceso y criterios de distribución}

La caracterización de un coste como indirecto, según la norma primera de las NODECOS (MINISDEF, 1998), viene dada por la existencia de factores de producción que, por afectar al proceso en su conjunto, impiden medir de forma técnica o económicamente precisa la incidencia de su consumo en el portador de coste final. La imputación de dichos costes al objetivo final de coste deberá practicarse empleando una base de distribución que sea fiel expresión de la relación causal entre los factores consumidos y los productos o servicios obtenidos. No obstante, Indra define los costes indirectos como aquéllos que no se pueden relacionar de una forma clara, evidente y directa con el contrato y están cargados a proyectos clasificados como de estructura e internos (Indra, 2006).

Lo más característico del sistema de costes diseñado por Indra, radica en el tratamiento de los costes indirectos. La distribución de éstos comienza por una agrupación funcional de los mismos y su localización en las distintas unidades de empresa (que desempeñan el papel, según la terminología de Indra, de "agrupadores de costes"5). En este sentido, la estructura organizativa de la em- presa fue utilizada a modo de agrupadores de costes indirectos para responder, no sólo a los requerimientos obligatorios de los organismos auditores, sino también a las necesidades de gestión de la propia empresa para conocer y formar una estructura de sus costes, revirtiendo esta obligación en una mejora de sus sistemas de información sobre sus propios costes.

En segundo lugar, los costes indirectos $^{6}$ de las unidades de empresa se distribuyen entre las actividades realizadas por cada unidad a otras, hasta acumularlos en los "mercados" (unidades de negocio de Indra). Los "mercados" representan el elemento conductor y acumulador del resto de costes indirectos. Seguidamente se procede, en el ámbito de los "mercados" que tienen contratos con el MINISDEF, al cálculo de los recargos de costes indirectos para su afectación al portador de coste (proyecto) vinculado al contrato en cuestión.

Sobre la base de la vinculación entre los costes indirectos y sus agrupadores de éstos que, en la mayoría de los casos, son tomados de la propia estructura orgánica de la empresa, se presentan las etapas para la distribución y acumulación final de todos los costes indirectos en los agrupadores de gastos comerciales también denominados "mercados" (Diagrama 3 ).

5 El objetivo principal de estos agrupadores de costes consiste en lograr una mayor claridad y control de los costes indirectos y, a su vez, en conseguir mayor facilidad para la imputación objetiva de los mismos a los diversos proyectos de los mercados que mantienen contratos con el MINISDEF.

"Gastos indirectos" en la terminología de Indra. 
a) Distribución de los costes indirectos de los agrupadores de "Gastos Generales".

Se trata de costes indirectos incurridos por las unidades de empresa que tienen la responsabilidad del buen funcionamiento de la compañía (Servicios Generales, Recursos Humanos, Vigilancia y Seguridad e Informática Interna) y cuyo objetivo es dar servicio al resto de la empresa. Estos costes indirectos de los agrupadores de gastos generales se distribuyen entre los agrupadores de gastos indirectos, gastos indirectos comerciales y mercados que tiene Indra en función de las claves de distribución recopiladas en el Cuadro 3.

b) Distribución de los costes indirectos de los agrupadores de "Gastos Indirectos".

Estos agrupadores recogen los costes indirectos de las unidades de empresa dedicadas a dar servicios corporativos y que, por definición, no tienen una relación directa con los "mercados" ni su fin está determinado por éstos. Los costes indirectos acumulados en cada uno de los siguientes agrupadores de gastos indirectos (tanto propios como recibidos de gastos generales) se asignan a cada "mercado" según las bases de distribución reflejadas en el Cuadro 4.

c) Distribución de los costes indirectos de los agrupadores de "Gastos Indirectos Comerciales".

Este tipo de costes indirectos son los incurridos por las unidades de la empresa que trabajan de manera directa y exclusiva para los "mercados", pero que jerárquicamente no pertenecen a ellos. Los costes indirectos comerciales registrados en los siguientes agrupadores de gastos indirectos comerciales (tanto propios como recibidos de gastos generales) se imputan a cada "mercado" en función de las bases de distribución contempladas en el Cuadro 5.

d) Los costes indirectos de los agrupadores de "Gastos Comerciales".

Son costes indirectos respecto a los proyectos facturables y corresponden a los incurridos en los agrupadores denominados "mercados". Estos costes están recogidos en proyectos de Estructura e Internos y corresponden a los mercados, teniendo la consideración de costes indirectos de cada mercado. Los agrupadores de los gastos comerciales se corresponden con los siguientes "mercados": Defensa; SIMSAM; Telecomunicaciones;

\section{Cuadro 3 \\ Distribución de los costes indirectos acumulados en los agrupadores de gastos generales}

\begin{tabular}{ll}
\multicolumn{1}{c}{\begin{tabular}{c}
\multicolumn{1}{c}{$\begin{array}{c}\text { Agrupador de Gastos } \\
\text { Generales }\end{array}$} \\
Informática Interna
\end{tabular}} & Base de Distribución \\
Servicios Generales & Dedicaciones \\
Vigilancia y Seguridad & Metros cuadrados \\
Recursos Humanos & Número de empleados en plantilla \\
\hline
\end{tabular}

Fuente: Elaboración propia a partir de Indra (2006). 


\section{Cuadro 4 \\ Reparto de los costes indirectos acumulados en los agrupadores de gastos indirectos}

\begin{tabular}{ll}
\hline \multicolumn{1}{c}{ Agrupador de Gastos Indirectos } & \multicolumn{1}{c}{ Base de Distribución } \\
\hline Órganos de Gobierno & Ventas \\
Económico & Ventas \\
Comunicación & Ventas \\
Desarrollo de Negocio Corporativo & Ventas \\
Análisis y Estrategia & Ventas \\
Financiero & Transferencias realizadas \\
Extraordinario & (No admisibles) \\
Interno Empresa & Específicamente \\
Dirección General de Cataluña & Ventas en mercados de Cataluña \\
Gestión Corporativa &
\end{tabular}

Fuente: Elaboración propia a partir de Indra (2006).

\section{Cuadro 5}

Distribución de los costes indirectos acumulados en los agrupadores de gastos indirectos comerciales

\begin{tabular}{ll}
\multicolumn{1}{c}{ Gastos Indirectos Comerciales } & \multicolumn{1}{c}{ Base de Distribución } \\
\hline Dirección General de Operaciones & Ventas \\
Planificación & Ventas \\
Centros de Competencia & Ventas \\
Dirección de Proyectos & Ventas \\
Marketing & Ventas \\
Iniciativas & Ventas \\
Calidad & Dedicaciones \\
Desarrollo, Planificación y Asignación & Dedicaciones \\
Centros de Producción & Dedicaciones \\
Internacional & Contratación internacional \\
Compras & Compras \\
Control de Mercados & A cada mercado sus gastos \\
Recursos Humanos de Mercados & A cada mercado sus gastos \\
Tecnología & Gastos de I+D \\
\hline
\end{tabular}

Fuente: Elaboración propia a partir de Indra (2006). 
Transportes y Tráfico; Administración Pública; Procesos Electorales; Finanzas y Seguros; Industria y Comercio; y Energía y Operadores.

Por tanto, como se puede apreciar, en los "mercados" o agrupadores comerciales se recolectan y acumulan todos los costes indirectos tanto propios de cada "mercado" (gastos comerciales) como recibidos de otras agrupaciones de costes indirectos (gastos generales, gastos indirectos y gastos indirectos comerciales) en función de las bases de reparto anteriormente citados.

Los "mercados" que tienen una relación más inmediata con los contratos que establece el MINISDEF con Indra son "Defensa" y SIMSAM" ${ }^{7}$. El "mercado" de defensa está organizado en siete divisiones ${ }^{8}$. A los efectos del cálculo diferenciado de los ratios, para establecer los recargos por concepto de costes indirectos deben ser imputados a los proyectos de defensa que tienen como cliente el MINISDEF, Indra estableció ciertas discriminaciones.

El "mercado" de defensa tiene dos sistemas de recargos distintos, uno que se puede considerar genérico aplicable a todas las divisiones excepto a la división de Sistemas de Gestión y otro aplicado exclusivamente a Sistemas de Gestión. Dentro del "mercado" de defensa, la división Sistemas de Gestión incluye a personal de Indra que presta sus servicios en los Cuarte- les Generales de los Ejércitos de Tierra y del Aire y de la Armada. Al desempeñar sus servicios en los Cuarteles Generales, y no en dependencias de la empresa, el consumo de recursos a imputar es distinto del resto de divisiones del "mercado" de defensa, lo cual motiva un tratamiento singular para esta división. El "mercado" de SIMSAM tiene otro sistema de recargos propio, distinto a los reseñados anteriormente para el "mercado" de defensa.

Ahora bien, la determinación de la cuantía de costes indirectos en las ofertas comerciales elaboradas por Indra para el MINISDEF, exige analizar la cuestión en tres niveles estrechamente relacionados:

a) Fijación de las agrupaciones de costes indirectos a emplear para la incorporación de los costes indirectos en el precio de la oferta.

Siguiendo lo establecido por la norma quinta de las NODECOS (MINISDEF, 1998) sobre condiciones de aplicación de los costes indirectos, se deben crear agrupaciones homogéneas, en el sentido de que sus actividades tengan la misma o similar relación causal o de utilidad respecto a los portadores u objetivos de costes con los que se relaciona.

Para responder a este requerimiento, Indra estableció los siguientes agrupadores de costes indirectos o "cost pool": agrupación de costes de indirectos

7 No obstante, y si bien se puede identificar el "mercado" SIMSAM con el agrupador SIMSAM, en el "mercado" de Defensa se recogen los costes indirectos de las unidades de empresa dedicadas al "mercado" de Defensa y Seguridad, así como también a la línea de actividad de Equipos Electrónicos de Defensa.

8 El "mercado" de Defensa incluye las divisiones: Mando y Control, Radares, Guerra Electrónica, Sistemas de Gestión, Proyecto European Fighter Aircraft, Sistemas y Post Venta. 
de fabricación, asociado al recargo de overhead; agrupación de costes por compras de materiales, ligado al recargo de manipulación de materiales; y agrupación de costes generales y de administración, vinculado al recargo de gastos generales y administración.

b) Determinación de la cuantía de las agrupaciones de costes indirectos.

Para cada uno de los agrupadores comerciales, Defensa, SIMSAM y Sistemas de Gestión, se calcula el importe de los costes indirectos de cada "cost pool" de la siguiente forma (Diagrama 5).

La agrupación de costes de cabecera, o de fabricación se forma a partir de los "gastos comerciales" propios más los que éstos reciben de "gastos generales", a los que se suman los "gastos indirectos comerciales" propios, a excepción del agrupador "Compras", y los recibidos de los "gastos generales" en una determinada proporción.

La agrupación de costes generales y administración se compone de los "gastos indirectos" propios y los recibidos de los agrupadores de "gastos generales". Además se deben incluir los costes procedentes de los proyectos de ofertas, en concreto, los de preparación y licitación y preparación de ofertas fallidas más sus recargos y los costes procedentes de los proyectos de $\mathrm{I}+\mathrm{D}$ no asociados a ningún contrato, que comprenden los gastos de I+D más sus recargos.

La agrupación de costes por manipulación de materiales se forma con los "gastos indirectos comerciales" del agru-

\section{Diagrama 5 \\ Agrupadores de costes indirectos en los mercados de Defensa y SIMSAM}

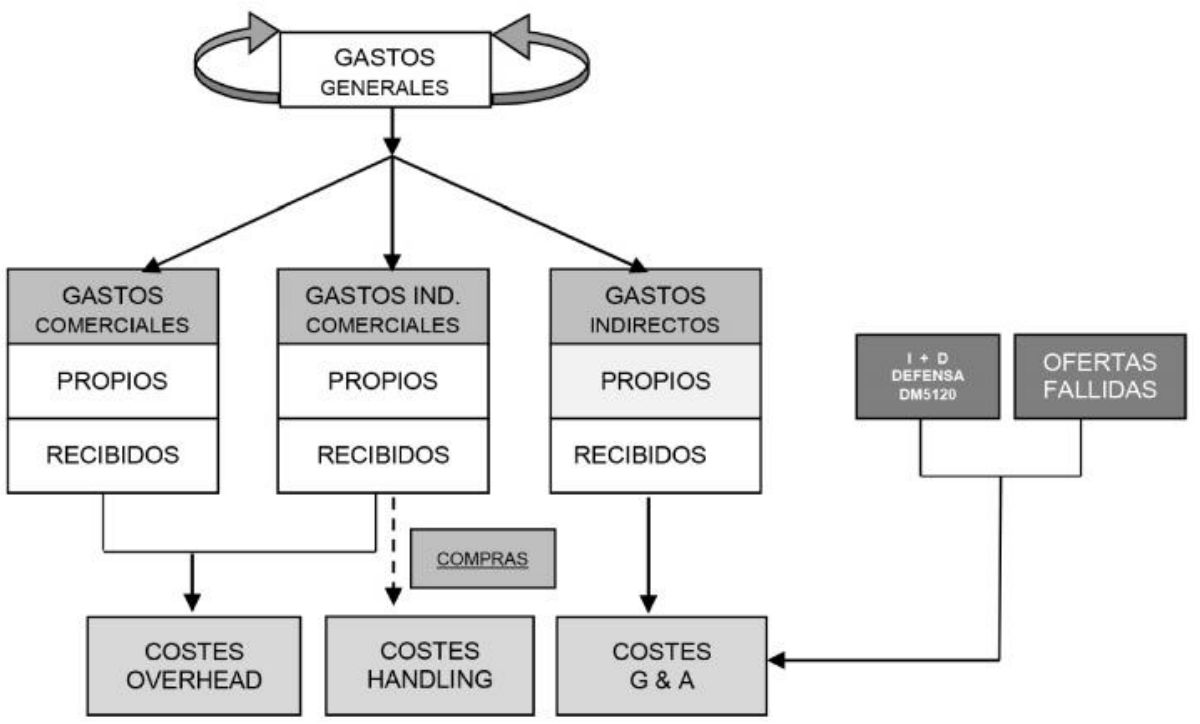

Fuente: Elaboración propia a partir de Indra (2004, 2005 y 2006). 
pador "Compras" y los costes que éste recibe de los agrupadores de "gastos generales". La Fijación de las bases de distribución a emplear en el cálculo de los recargos.

El requisito fundamental para la elección de una base de distribución, según la norma quinta de las NODECOS (MINISDEF, 1998) radica en que ésta sea representativa de la actividad gestionada o supervisada. La base de reparto debe estar integrada por todos los elementos más significativos $\mathrm{y}$, aunque parece optarse por la elección del número de horas empleadas o el coste de la mano de obra directa consumida, se deja la puerta abierta a la elección de otras que se consideren más idóneas para recoger el grado de variabilidad de los costes de cada agrupación de costes indirectos específica.

Indra para el cálculo del recargo de overhead (Ecuación 1) utiliza como base de distribución el consumo de mano de obra directa de los proyectos calificados como de costes directos (facturables, ofertas, e l+D).

\footnotetext{
Recargo Agrupación de costes de cabecera de Overhead $=\frac{\text { Consumo de mano de obra directa de }}{\text { Con }}$ proyectos directos
}

Para el cálculo del recargo de manipulación de materiales (handling) se distribuye la agrupación de costes por compra de materiales en función del consumo de materiales, y subcontratación de los proyectos de costes directos (Ecuación 2).

\footnotetext{
Recargo = Agrupación de costes por compras de Handling $=$ Consumo demateriales y subcontratación deproyectos directos
}

Por último, para el cálculo del recargo de gastos Generales y Administración (G\&A), la empresa toma como base de distribución la suma del coste directo total de los proyectos facturables más los recargos de costes indirectos de fabricación y el de manipulación de materiales (Ecuación 3).

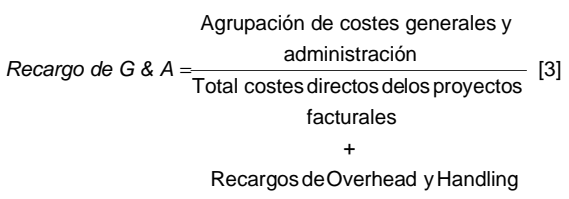

En el proceso de cálculo de los recargos para la imputación de los costes indirectos se utilizan coeficientes preestablecidos de acuerdo con la norma quinta de NODECOS (MINISDEF, 1998). Es decir, Indra estima los recargos en función de los datos registrados en periodos anteriores y dichos coeficientes se actualizan y revisan una vez al año. Se suelen emplear técnicas estadísticas de regresión lineal para su cálculo, utilizando una ecuación de curva de ajuste logarítmica que permite obtener el valor esperado en el siguiente año. Esta información es analizada, junto a las tasas horarias de la mano de obra directa, por el GEC y, una vez informada positivamente por él, es utilizada por los órganos del MINISDEF en la elaboración del presupuesto, negociación y fijación de los precios de los contratos con Indra.

\subsection{Los costes financieros}

La norma octava de las NODECOS (MINISDEF, 1998) reconoce el coste financiero como un elemento de coste más de los contratos que las empresas pueden incluir como componente del precio de las ofertas para el MINISDEF. 
Dado que la naturaleza del coste financiero es diferente a la de los costes indirectos descritos, y la norma octava exige su presentación en la oferta del contratista como partida independiente del resto de costes, el estudio de los recargos financieros se realiza de forma separada del resto de recargos de gestión.

La cuantificación e imputación de los costes financieros, en su consideración como coste afecto a la actividad productiva, se calcula en términos de costes de oportunidad. Indra se adapta a lo dispuesto en la norma octava y establece en su sistema de cálculo de costes dos tipos de recargos financieros para las siguientes clases de costes financieros: a) costes financieros del capital invertido en el inmovilizado afecto a la actividad productiva (FCCOM, por las siglas en inglés Facilities Capital Cost of Money), y b) costes financieros asociados a los recursos invertidos en factores corrientes, componentes del circulante y afectos a las operaciones relacionadas con el MINISDEF.

a) Costes financieros del capital invertido en el inmovilizado afecto a la actividad productiva.

Para el cálculo del recargo FCCOM, Indra, cumpliendo las condiciones previstas en la norma octava de las NODECOS (MINISDEF, 1998) utiliza los valores netos contabilizados en su inmovilizado material e intangibles (este último con algunas excepciones) que sean utilizados en la actividad productiva normal del negocio.

Una vez realizada la distribución de los inmovilizados en cada "mercado", el total de activos asignados se utiliza para calcular el coste financiero del capital invertido en el inmovilizado afecto a la actividad productiva (FCCOM). El cálculo del recargo FCCOM se realizaría según la Ecuación 4:

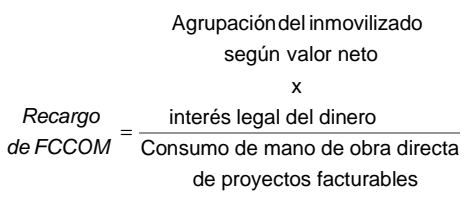

b) Costes financieros asociados a los recursos invertidos en factores corrientes, componentes del circulante.

Una observación importante sobre estos recargos (Periodo Medio de Maduración y Periodo Medio de Cobro), es la reducida aplicación real y efectiva de estos recargos como componentes de coste en los contratos del MINISDEF. Las posibles razones de este tratamiento pueden situarse en la gestión de los almacenes de materiales que tiene la empresa, tendentes a su reducción o consideración mínima y al hecho de la consideración de cliente habitual y estratégico que tiene el MINISDEF para Indra.

\subsection{El beneficio}

El cuarto elemento en que se descompone el precio lo constituye el beneficio. Según se señala en los modelos de pliegos de cláusulas administrativas particulares establecidos por el MINISDEF para la adjudicación de contratos por el procedimiento negociado, este elemento debe constar en la oferta presentada por las empresas interesadas en contratar con el MINISDEF. 
En España no existe una fórmula predeterminada para el cálculo del margen de beneficio aplicable a este tipo de contratos. Si bien las directrices teóricas generales del MINISDEF sobre la determinación de márgenes y beneficios de los contratos públicos inciden principalmente en las circunstancias de riesgo e innovación y ahorro de costes en la ejecución, en la práctica el juego de intereses entre ambas partes (Indra y MINISDEF) parece desvirtuar tales directrices. A nuestro juicio, el protagonismo en la determinación tanto del precio final del contrato como del beneficio asociado al mismo, parece residir en el MINISDEF.

Los órganos de contratación tienen una limitación económica y presupuestaria para obligarse con terceros representada por el importe económico del crédito disponible, asignable y adecuado para cubrir la necesidad declarada por la Administración Pública. La misma fija el precio máximo de adjudicación del contrato y, por tanto, es el órgano de contratación del MINISDEF quien, con sus créditos, lo fija inicialmente. De igual manera, los órganos de contratación establecen en cada contrato las fórmulas de contratación y con ello influyen en la forma de fijar el precio de aquél (precio fijo, precio provisional, precio máximo con incentivos por ahorros de costes, reembolso de costes más un porcentaje de beneficio $u$ otros).

Tampoco debe olvidarse que en la negociación entre empresa y MINISDEF son objeto de discusión tanto el precio del contrato como el porcentaje de beneficio a recibir por la empresa. Por tanto, y según la modalidad de contratación empleada, la determinación del beneficio y, en consecuencia, la cuantificación total del precio del contrato puede que no quede determinada hasta la realización de una auditoría de costes incurridos de la cual pueden derivarse ajustes económicos para Indra.

La gran mayoría de los contratos celebrados entre el MINISDEF e Indra se realizaron bajo la modalidad del precio cierto del contrato, permitiendo a la empresa tener un mayor control sobre sus costes y así maximizar su propio margen de beneficio. No obstante, actualmente los órganos de contratación del MINISDEF están aplicando nuevas fórmulas que tratan de incentivar el beneficio de las empresas en función de los riesgos asumidos en los proyectos, así como del ahorro de costes logrado en su ejecución. Estas nuevas fórmulas de fijación del precio de los contratos suponen un incentivo para la mejora en la gestión y desarrollo de los proyectos lo cual, en última instancia, debe traducirse en una reducción de los costes inicialmente previstos $y$, en consecuencia, en una mejora de los márgenes de beneficio de los proyectos acometidos.

\section{Conclusiones}

El sometimiento de los contratistas del sector de defensa a la normativa sobre cálculo de costes y a las auditorías de los contratos impuesta por el MINISDEF en sus relaciones contractuales, tuvo como resultado la adopción por parte de aquellos de un modelo de sistema de costes completos basado en las directrices de las NODECOS (MINISDEF, 1998).

Tradicionalmente, Indra Sistemas S.A. implementaba en sus sistemas de información contable el modelo de asignación de costes parciales, en su modalidad de costes directos. Sin embargo, la 
configuración por parte de la referida empresa del precio de los contratos de acuerdo con la normativa sobre costes establecida por el MINISDEF, obligó a la misma a prestar una mayor atención al control y al tratamiento contable de los costes indirectos. En la práctica, ello implicó una mejora de la información relativa al proceso de generación de costes en la empresa y, por ende, del control de aquellos asociados a los diferentes contratos y del seguimiento de su ejecución.

El estudio empírico llevado a cabo sobre las fases del proceso de cálculo de los costes de los contratos seguido por Indra Sistemas, S.A. en sus relaciones contractuales con el MINISDEF, representa una guía orientativa para otras empresas que en un futuro puedan contratar con dicho departamento. Al mismo tiempo, arroja luz sobre la mecánica de cálculo seguida en un ámbito económico no siempre caracterizado por su transparencia informativa en este terreno.

Del estudio realizado se puede deducir que el sistema de costes empleado por Indra se encuentra muy próximo a la estructura de costes que presentan los sistemas orgánicos, siendo lo más peculiar y, por tanto, lo más destacable, el tratamiento otorgado a los costes indirectos. Las unidades de la empresa y los agrupadores determinan la clase de coste indirecto que en él se incurre. La utilización de su estructura orgánica para la localización, agrupación y acumulación de los costes indirectos en las unidades de empresa denominadas "mercados", a partir de los cuales se calculan los recar- gos de gestión en concepto de costes indirectos, es lo que caracteriza su sistema de costes. En este sentido, de los elementos que configuran el precio del contrato, el cálculo y la imputación de los costes indirectos constituyen la principal dificultad que tienen que afrontar las empresas para la determinación objetiva y fiable del coste de los contratos, de acuerdo con las disposiciones de las NODECOS (MINISDEF, 1998).

Al no existir en España una norma específica que regule la determinación del beneficio "razonable", ni una fórmula predeterminada para el cálculo del margen del beneficio aplicable a los contratos públicos de defensa, éste elemento representa el componente del precio de un contrato menos objetivo en su estimación. Las nuevas fórmulas de contratación y las nuevas formas de fijar el precio de los contratos demandan una regulación específica de los márgenes de beneficio que aporte mayor objetividad y transparencia en su cálculo, valorando y ponderando las diversas circunstancias que concurren en cada contrato en particular.

Por último, se considera que la metodología empleada por el MINISDEF para la evaluación de costes en sus contratos, con independencia de las implicaciones que pudieran tener en los sistemas de contabilidad de gestión de las empresas auditadas para adaptarse a ese marco normativo, contribuye a aumentar la transparencia de la gestión realizada por los órganos de contratación de éste y la legitimación del gasto público en defensa ante los contribuyentes. 


\section{Referencias Bibliográficas}

Abzug, Rikki y Mezias, Stephen J. (1993). The fragmented state and due process protections in organizations: the case of comparable worth. Organization Science, Vol. 4, № 3, pp. 433-453.

Aguado Romero, José (2009). El control económico de las adquisiciones públicas en materia de defensa. Granada. Editorial de la Universidad de Granada.

Aguado Romero, José y Zafra Gómez, José Luis (2011). Las normas de costes aplicables en el análisis de costes y precios de los contratos de defensa en España. Presupuesto y Gasto Público, № 65/2011, España, Instituto de Estudios Fiscales, pp. 7-26.

Al-Omiri, Mohammad y Drury, Colin (2007). A survey of factors influencing the choice of product costing systems in UK organizations. Management Accounting Research, Vol. 18 (3), pp. 399-424.

Araujo Pinzón, Pedro (2003). Nuevo Institucionalismo, Teoría de la estructuración y cambio en los sistemas y prácticas de contabilidad de gestión: Teorías y métodos de investigación. Revista Española de Financiación y Contabilidad, Vol. 32. № 118, España, pp. 693-724.

Arviso, Beverly (2006). Surviving a DCAA audit. Business Credit, 108, p. 29.

Bescos, Pierre-Laurent (2003). New Tasks for Management Accounting. Management Accounting Section Research and Case Conference, San Diego, California, January, pp.10-11.

Burns, James E. (2000). The dynamics of accounting change: Interplay between new practices, routines, institutions, power and politics. Accounting, Auditing and Accountability Journal, Vol. 13, № 5, pp. 566-596.

Carmona, Salvador; Ezzamel, Mahmoud y Gutiérrez, Fernando (1998). Towards and institutional analysis of changes in accoun- ting in the Royal Tobacco Factory of Seville. The Accounting Historian Journal, Vol. 25, No 1, pp. 115-147.

Carmona, Salvador y Macías, Marta (2001). Institutional pressures; monopolistic conditions, and the implementation of early cost management practices: The Case of the Royal Tobacco Factory of Seville (1820-1887). Abacus, Vol. 37, № 2, pp.139-165.

Carruthers, Bruce G. (1995). Accounting, Ambiguity, and the New Institutionalism.

Accounting, Organizations and Society, Vol. 20 (4), pp. 313-328.

Covaleski, Mark A., Dirsmith, Mark. W., y Michelman, Jeffrey E. (1993). An institutional theory perspective on the DRG framework, case-mix accounting systems and health-care organizations. Accounting, Organizations and Society, Vol.18, № 1, pp. 65-80.

Currie, David (2011). Review of single source pricing regulations. Disponible en: http://www.mod.uk/NR/reiew_singe_s ource_pricing_regulation. (fecha de consulta: 28/03/2012).

Dirección General de Armamento y Material (2011). La industria española de defensa. Ministerio de Defensa, España. Disponible en: http://10.7.81.76/webintranetdgam/red.htm. (fecha de consulta: 26/10/2011).

Escobar Pérez, Bernabé y Lobo Gallardo, Antonio (2002). Implicaciones teóricas y metodológicas de la evolución de la investigación en contabilidad de gestión. Revista Española de Financiación y Contabilidad, Vol. 31, № 111, España, pp. 245-286.

Fonfría Mesa, Antonio y Correa Burrows, Paulina (2010). Effects of military spending on the profitability of spanish defence contractors. Defence and Peace Economics, Vol. 21, No 2, pp. 177-192.

Gil, José Antonio (2002). Reestructuración de la industria de defensa en España, en 
La reestructuración de las industrias de defensa, Minerva Ediciones, pp. 53-76.

Granlund, Markus (2001). Towards explaining stability in and around management accounting systems. Management Accounting Research, Vol. 12, pp. 141-166.

Indra (2004). Recargos ofertas comerciales presupuesto 2004. Trabajo no publicado, Madrid.

Indra (2005). Informe de análisis económico de la propuesta de costes horarios y recargos de gestión para el año 2005 remitida por la empresa Indra Sistemas S.A. Trabajo no publicado, Madrid.

Indra (2006). NODECOS en Indra. Sistema para el cálculo de recargos, Gerencia de Control de Gestión. Trabajo no publicado, Madrid.

Kirat, Thierry; Bayon, Denis y Blanc, Helene (2003). Maîtriser les coûts des programmes d'armement: une analyse comparative de la réglementation des marchés industriels d'armement en France, au Royaume-Uni et aux Etats-Unis. Observatoire économique de la défense, La Documentation française. Paris.

Ministerio de Defensa (1998). Orden del Ministerio de Defensa 238/1998, de 15 de octubre, sobre presentación y auditoría de ofertas y normas sobre los criterios a emplear en el cálculo de costes de determinados contratos de suministro, de consultoría y asistencia y de los servicios del Ministerio de Defensa que se adjudiquen por el procedimiento negociado (NODECOS) (BOE № 258, de 28 de octubre), España.
Rogerson, William P. (1989). Profits regulation of defense contractors and prizes for innovation. Journal of Political Economy, Vol. 97, № 6, pp. 1284-1305.

Scapens, Robert W. (1994). Never mind the gap: towards an institutional perspective on management accounting practice. Management Accounting Research, Vol. 5, pp. 301-321.

Scott, W. Richard (1995). Institutions and Organizations. London, Sage Publications.

Secretaria de Estado de la Defensa (2007). Instrucción 128/2007, de 16 de octubre, por la que se aprueba el procedimiento para la prestación de los servicios de análisis de costes y precios en el ámbito del MINISDEF (Boletín Oficial de Defensa № 212, de 30 de octubre), España.

Selto, Frank H. y Widener, Sally K. (2004). New management accounting research: Insights from practice. Advances in Management Accounting, Vol. 12, pp. 1-36.

Smith, Ronald Patrick (1990). Defence procurement and industrial structure in the UK. International Journal Industrial Organization, Vol. 8, pp. 185-205.

Stockholm International Peace Research Institute (2012). SIPRI Military Expenditure Database. Disponible en: http://www.sipri.org/database/milex. [fecha de consulta: 2012, Abril 10].

Suchman, Mark C. (1995). Managing legitimacy: Strategic and institutional approaches. Academy of Management Review, Vol. 20, № 3, pp. 571-610.

Yin, Robert K. (2003). Case study research: design and methods. California, $3^{\text {a }}$ Edition Thousand Oaks, Sage Publications. 